Psychological Medicine, 1978, 8, 177-180

Printed in Great Britain

\title{
EDITORIAL
}

\section{Pharmacokinetics and psychotropic drugs ${ }^{1}$}

The current wave of interest in pharmacokinetics of psychotropic drugs has its origins in the writings of Brodie in the years between 1960 and 1970 (for review see Brodie, 1967). Strictly speaking, the science of pharmacokinetics is concerned with rates of transfer of drug molecules within the body. Less strictly, it is the study of drug concentrations in plasma. A lifetime of research in drug analysis, and in the study of drug absorption, metabolism and excretion, had led Brodie to believe that many hitherto poorly controlled drug responses could be brought under control by changing the plasma levels of the drugs concerned. There seemed no reason why this idea should not apply equally to drugs acting on the central nervous system as to drugs affecting other systems, in spite of the bloodbrain barrier, and so Brodie encouraged a number of workers to commence investigations of tranquillizers and antidepressants in this light (Curry \& Brodie, 1967; Hammer \& Brodie, 1967; Garattini et al. 1973). In the meantime, other workers were investigating lithium (Schou, 1969). However, in spite of more than 10 years of this work, and in spite of the fact that pharmacokinetic control has been a major factor in the use of lithium, fundamental misunderstandings remain. We are repeatedly asked: 'What should we measure, and with what methods?' 'What is the relation with effect?' 'Is there a clinically desirable concentration?'

Analytical methods are the oldest problem. Without good methods the work could not start. The major analytical achievements relevant to this work were the application of isotope derivative analysis to tricyclic antidepressants (Hammer \& Brodie, 1967), and of gas-chromatography with electron capture detection to phenothiazines, and to benzodiazepines (Curry, 1968; Garattini et al. 1973). Later, combined gas-chromatography/mass spectrometry came prominently into the picture. In the hands of careful workers, these techniques now give reliable data. Provided reasonable precision and accuracy are demonstrated to have been achieved in any laboratory for a specific compound, the exact details of the method used in that laboratory are not important. For instance, at an absurd level, the shape of the test tubes does not matter if the method is proved adequate. However, authors have been able to claim significant modifications by doing obvious things, such as doubling the sample size to double sensitivity. Even so, the complexities are great. For example, chlorpromazine is partially converted to chlorpromazine $N$-oxide in the body. Reduction of the metabolite, present in plasma, back to chlorpromazine apparently occurs on storage of samples, and during analytical work-up. Unless strenuous efforts are made to prevent this reduction, inflated chlorpromazine data are obtained. So the chlorpromazine content of the sample may appear to increase on storage or, at best, appear to be erratic, Now, reduction in assay results on storage is all very well, but an increase? I cite this example to demonstrate the need for detailed knowledge of the chemistry involved in such work. Each group of workers should be asked for details of precision and accuracy of methods as used in its own laboratories.

Another point of relevance to the assays is that authors are not always specific regarding fluids measured. 'Blood levels' and 'plasma levels', to say nothing of 'serum levels', are still used as interchangeable terms. Additionally, centrifugation conditions for plasma are rarely checked to determine whether any haemolysis or cell contamination occurs. Haemolysis can increase the chance of a distorted assay, because of a difference in drug concentrations in the fluid of red cells and of plasma. If the drug is localized in red cells, haemolysis inflates the plasma concentration. If the drug fails to penetrate red cells, haemolysis reduces the plasma concentration.

Pharmacokinetic studies are the best way of studying the pharmacokinetic phenomena of absorption and elimination in their own right and, related to this, of obtaining data on such matters as bioavailability and drug interactions. They also assist detection of non-compliance in tablet-taking, although, for complex reasons associated with metabolism of oral doses during the first-pass through

1 Address for correspondence: Dr S. H. Curry, The London Hospital Medical College, Turner Street, London E1 2AD. 
the liver, urinary excretion of metabolites may be a better guide to this particular problem. However, it is in relation to effect that the subject comes into its own. In this regard there is a major problem of what to include. Most of the psychotropic drugs are converted to many metabolites. Some of these metabolites are polar and inactive. Some are rapidly removed from the body once formed and therefore not detected in plasma, in accord with the normal function of drug metabolism - to make excretion possible. Others have significant activity, for example, desipramine as a metabolite of imipramine. Investigators are faced with the need to categorize those metabolites which appear in plasma as 'active' or 'inactive' and this must usually be done with only in vitro work as a guide, because direct human studies with drug metabolites are rarely possible. When such studies are possible, the data are often equivocal. For example, chlorpromazine sulphoxide 'failed' in clinical studies, and a habit is developing of designating this compound as inactive. However, it has oneseventh the sedative activity of chlorpromazine and the antipsychotic effect was not really assessed in the studies concerned (Davidson et al. 1957; Sakalis et al. 1973).

When more than one apparently active compound is found in plasma, for example, imipramine and desipramine following imipramine administration, the dilemma is whether to relate effects to the two (or more) compounds separately, or to sum the various components, or to try weighting for the differential potencies of the compounds (Moody et al. 1967). For example, if compound A (potency one unit) is converted to compound $B$ (potency 0.5 units) and $A$ is found at $50 \mathrm{ng} \mathrm{ml}^{-1}$ while $B$ is at $30 \mathrm{ng} \mathrm{ml}^{-1}$, do we have $(1 \times 50)+(0.5 \times 30)=65 \mathrm{ng} \mathrm{ml}^{-1}$ equivalents of drug? Such contortions seem excessive. Another device is the use of ratios, such as the one creeping into chlorpromazine literature (Mackay et al. 1974; Sakalis et al. 1977):

\section{chlorpromazine + 7-hydroxychlorpromazine \\ chlorpromazine sulphoxide}

This breaks all the rules, as the top line allegedly comprises active compounds, but with different potencies determined in human (chlorpromazine) and animal models (7-hydroxychlorpromazine), while the bottom line includes an allegedly inactive compound (but see earlier). Furthermore, the ratio is of two variables which are not independent, as each molecule of chlorpromazine can only be converted to one of the two metabolites (it can of course be converted to yet other metabolites). Statistically, if the concentrations of each of the compounds incorporated into such ratios in different groups of subjects are not significantly different, then any apparent significance in contrived ratios is quite fallacious. If one figure, for example chlorpromazine sulphoxide in the above ratio, is significantly different in different groups of patients, then the ratio merely confirms that this is the case, without providing any new information.

This is in fact the case with chlorpromazine. In studies of long-term patients, chlorpromazine and 7-hydroxychlorpromazine have failed to show significant differences in groups of patients categorized as responding well, indifferently, or not well. However, poor responders show elevated chlorpromazine sulphoxide levels. They also receive higher doses. So it seems that long-term patients are prescribed doses which generate common maximum plasma chlorpromazine and 7-hydroxychlorpromazine concentrations, limited by tolerability of unwanted effects, with the excess being converted to chlorpromazine sulphoxide. The clinical outcome then depends very much on non-pharmacological factors. Although there is an inverse relation between success of treatment and chlorpromazine sulphoxide concentration, there is no suggestion of the sulphoxide being psychotoxic.

Interest in the metabolites of chlorpromazine has been immense, but it now seems that only 7-hydroxychlorpromazine and demonomethylchlorpromazine are of great importance. However, there is a divergence of views. In acute patients with chlorpromazine in their plasma, in controlled studies, response occurs with negligible amounts of the metabolites present (Curry \& Evans, 1976). In chronic patients, in uncontrolled studies, significant amounts of the metabolites are present (Mackay et al. 1974). So it seems that those metabolites which have appreciable activity (shown in animals of course, and below that of chlorpromazine itself) contribute to the action of chlorpromazine when present, but are not essential to the action of chlorpromazine. 
With both chlorpromazine and tricyclic antidepressants, various other observations have been made regarding clinical outcome in relation to plasma levels. Over narrow ranges, there is no correlation to plasma levels. Over wide ranges, $U$-shaped relations are seen, with low concentrations inadequate, and high concentrations adverse (Åsberg et al. 1971; Curry, 1976). Again, it is not suggested that the drugs are psychotoxic in high concentrations. The $U$-shaped relation is observed using global rating scales, which take into account unwanted effects of drugs which occur when concentrations are excessive. This has been shown repeatedly with tricyclics and phenothiazines, and also with benzodiazepines and lithium. Some rules for any clinical relationship appear to be:

(1) The relation will depend heavily on the type of patients and the mode of assessment.

(2) The clinically useful range will be wide.

(3) The relation will be more obvious when individuals are studied repeatedly than when a population is sampled for a correlation.

(4) Too fine a relationship should not ke expected.

This appears to be the basis for the diflerence of opinion among those investigating tricyclic antidepressants, a difference of opinion which has led to heated exchanges at meetings. Nobody need be making incorrect observations. Even so, rather than using clinical rating, should we be using pharmacological or biochemical measures? There is as yet no answer to this. With several examples peripheral effects, such as pulse rate and pupil size, correlate better with plasma levels than does clinical rating, but these effects do not particularly correlate with clinical rating. Also, much interest has been centred on prolactin and platelets, but not as measures of psychosis in any way (Sakalis et al. 1972; Loga et al. 1975; Boullin et al. 1975; Kolakowska et al. 1975).

So is clinical monitoring useful? When the analyst is competent, and a suitable person is available to interpret data, monitoring picks out underdosed and overdosed patients, neither of whom will always be apparent on clinical grounds alone. This can te a help in individualizing dosing regimes. Arguably, clinical monitoring should therefore always te available to the prescriber for investigation of problem cases, as Brodie's idea appears to hold true, that drug treatment in man is better controlled if pharmacokinetic variability is compensated with individually tailored dosage regimes. However, clinical monitoring costs money.

STEPHEN H. CURRY

\section{REFERENCES}

Åsberg, M., Cronholm, B., Sjöqvist, F. \& Tuck, D. (1971). Relationship between plasma level and therapeutic effect of nortriptyline. British Medical Journal iii, 331-334.

Boullin, D. J., Woods, H. F., Grimes, R. P. J., GrahameSmith, D. G., Wiles, D. H., Gelder, M. G. \& Kolakowska, T. (1975). Increased platelet aggregation responses to 5-hydroxytryptamine in patients taking chlorpromazine. British Journal of Clinical Pharmacology 2, 29-35.

Brodie, B. B. (1967). Physicochemical and biochemical aspects of pharmacology. Journal of the American Medical Association 202, 600-609.

Curry, S. H. (1968). Determination of nanogram quantities of chlorpromazine and some of its metabolites in plasma using gas-liquid chromatography with an electron-capture detector. Analytical Chemistry 40, 1251-1255.

Curry, S. H. (1976). Gas-chromatographic methods for the study of chlorpromazine and some of its metabolites in human plasma. Psychopharmacology Communications 2, $1-15$.

Curry, S. H. \& Brodie, B. B. (1967). Estimation of nanogram quantities of chlorpromazine (CPZ) in plasma using gasliquid chromatography (GLC) with an electron-capture detector. Federation Proceedings 26, 761.

Curry, S. H. \& Evans, S. (1976). A note on the assay of chlorpromazine $N$-oxide and its sulphoxide in plasma and urine. Journal of Pharmacy and Pharmacology 28, 467-468.

Davidson, J. D., Terry, L. L. \& Sjoerdsma, A. (1957). Action and metabolism of chlorpromazine sulfoxide in man. Journal of Pharmacology and Experimental Therapeutics $121,8-12$.

Garattini, S., Marcucci, F., Morselli, P. L. \& Mussini, E. (1973). The significance of measuring blood levels of benzodiazepines. In Biological Effects of Drugs in Relation to their Plasma Concentrations (ed. D. S. Davies and B. N. C. Prichard), pp. 211-225. Macmillan: London.

Hammer, W. \& Brodie. B. B. (1967). Application of isotope derivative technique to assay of secondary amines. Estimation of desipramine by acetylation with $\mathrm{H}^{3}$ acetic anhydride. Journal of Pharmacology and Experimental Therapeutics 157, 503-508.

Kolakowska, T., Wiles, D. H., Gelder, M. G. \& McNeilly, A. S. (1975). Correlation between plasma levels of prolactin and chlorpromazine in psychiatric patients. Psychological Medicine 5, 214-216.

Loga, S., Curry, S. H. \& Lader, M. H. (1975). Interactions of orphenadrine and phenobarbitone with chlorpromazine: plasma concentrations and effects in man. British Journal of Clinical Pharmacology 2, 197-208.

Mackay, A. V. P., Healey, A. F. \& Baker, J. (1974). The relationship of plasma chlorpromazine to its 7-hydroxy and sulphoxide metabolites in a large population of chronic schizophrenics. British Journal of Clinical Pharmacology 1 , 425-430.

Moody, J. P., Tait, A. C. \& Todrick, A. (1967). Plasma levels of imipramine and desmethylimipramine during therapy. British Journal of Psvchiatry 113. 183-193. 
Sakalis, G., Curry, S. H., Mould, G. P. \& Lader, M. H. (1972). Physiologic and clinical effects of chlorpromazine and their relationship to plasma level. Clinical Pharmacology and Therapeutics 13, 931-946.

Sakalis, G., Chan, T. L., Gershon, S. \& Park, S. (1973). The possible role of metabolites in therapeutic response to chlorpromazine treatment. Psychopharmacologia (Berlin) 32, 279-284.

Sakalis, G., Chan, T. L., Sathananthan, G., Schooler, N.,
Goldberg, S. \& Gershon, S. (1977). Relationships among clinical response, extrapyramidal syndrome and plasma chlorpromazine and metabolite ratios. Communications in Psychopharmacology 1, 157-166.

Schou, M. (1969). Lithium: relation between clinical effect of the drug and its absorption, distribution and excretion. In The Present Status of Psychotropic Drugs (ed. A. Cerlett and J. J. Bove), pp. 120-122. Excerpta Medica: Amsterdam. 